\title{
Tunable THz Signal Generation and Radio- over-Fiber Link based on an Optoelectronic Oscillator-driven Optical Frequency Comb
}

\author{
G.K.M. Hasanuzzaman ${ }^{1}$, Student Member, IEEE, Haymen Shams ${ }^{2}$ Member, IEEE, Cyril C. Renaud ${ }^{2}$, \\ Senior Member, IEEE, John Mitchell ${ }^{2}$, Senior Member, IEEE, Alwyn J. Seeds ${ }^{2}$, Fellow, IEEE and \\ Stavros Iezekiel ${ }^{1}$, Senior Member, IEEE
}

\begin{abstract}
We propose and demonstrate experimentally a photonic $\mathrm{THz}$ signal generation technique based on a tunable optoelectronic oscillator (OEO), and its application in a radio over fiber (RoF) link. The OEO's frequency is tuned by varying the bandwidth of a tunable optical bandpass filter (TOBF) that is cascaded with a phase modulator (PM). The resulting tunable microwave photonic filter is used to generate OEO oscillations from $6.58 \mathrm{GHz}$ up to $18.36 \mathrm{GHz}$ (with a phase noise of $\leq-103$ $\mathrm{dBc} / \mathrm{Hz}$ at $10 \mathrm{kHz}$ offset from the carrier frequency). The $\mathrm{OEO}$ is subsequently used to drive an optical comb, generating 22 comb lines with a frequency spacing of $17.33 \mathrm{GHz}$ covering a bandwidth of $360 \mathrm{GHz}$ within a $20 \mathrm{~dB}$ envelope. By selecting two optical comb lines with a wavelength selective switch (WSS) and beating them in a uni-traveling carrier photodiode (UTC-PD), THz signals are generated at $101.5 \mathrm{GHz}$ and $242.6 \mathrm{GHz}$ with phase noise of $-90 \mathrm{dBc} / \mathrm{Hz}$ and $-78 \mathrm{dBc} / \mathrm{Hz}$, respectively at $10 \mathrm{kHz}$ offset from carrier frequency. Tunable mm-wave and $\mathrm{THz}$ signals can be generated either by changing the OEO oscillation frequency or the selected comb lines. Using the OEO driven OFCG, we implemented a RoF link at $242.6 \mathrm{GHz}$ with a data rate of 24 Gbps over a wireless distance of $30 \mathrm{~cm}$ and with a bit error rate (BER) below the hard decision forward error correction (FEC) limit of 3.8 $\times 10^{-3}$. This method allows the creation of an allphotonic frequency reconfigurable $\mathrm{THz}$ signal generator and RoF system.
\end{abstract}

Index Terms - Optoelectronic oscillator, Side mode suppression, Infinite impulse response, Allan deviation, Microwave Photonics.

\section{INTRODUCTION}

$\mathrm{R}$ adio over fiber (RoF) technology enables seamless media conversion between radio and optical signals in a fiberwireless link and plays a key role in reducing the offset

This project has received funding from the European Union's Horizon 2020 research and innovation programme under the Marie Skłodowska-Curie grant agreement No. 642355. G.K.M. Hasanuzzaman is grateful to the Department of Electronic and Electrical Engineering, University College London (UCL) for the honorary research associate position.

G.K.M. Hasanuzzaman and Stavros Iezekiel are with the Microwave Photonics Research Laboratory, EMPHASIS Research Centre, University of Cyprus, Nicosia, 1678, Cyprus (e-mail: ghasan01@ucy.ac.cy).

Haymen Shams, Cyril C. Renaud, John Mitchell and Alwyn J. Seeds are with the Department of Electronic and Electrical Engineering, University College London, Torrington Place, London, WC1E 7JE, UK. between wireline and wireless data rates. Consequently, RoF with high capacity fiber-wireless links operating at the $\mathrm{mm}$ wave and $\mathrm{THz}$ band is being considered in mobile backhauling and front-hauling for the 5G era and beyond [1], [2]. Other applications include the enhancement of network resilience against disasters [3], the last mile access network where deployment of the optical network is difficult or not economical, and non-telecom applications, such as linear cell radar systems [4]. In all these applications, the generation of low phase noise mm-wave and $\mathrm{THz}$ waves is a crucial function.

Since the $\mathrm{THz}$ region is positioned between the microwave and infrared, there exist two enabling technologies for $\mathrm{mm}$ wave and $\mathrm{THz}$ signal generation, namely electronics and photonics [5], [6]. Photonic-assisted approaches to generate $\mathrm{mm}$-waves and $\mathrm{THz}$ waves are of interest because they enable distribution of high-quality carriers to a remote site via lowloss optical fiber. Of the photonics-based approaches to microwave and $\mathrm{mm}$-wave signal generation, the optoelectronic oscillator (OEO) is particularly attractive because it can generate low phase noise oscillation without any reference RF signal [7]; a state-of-the-art X-band OEO has a phase noise as low as $-163 \mathrm{dBc} / \mathrm{Hz}$ at an offset frequency of $6 \mathrm{kHz}$ for a 10 $\mathrm{GHz}$ output [8]. The highest reported OEO oscillation frequency is $60 \mathrm{GHz}$ [9]. However, there is interest in wireless links operating in the W-band $(75-110 \mathrm{GHz})$ and in the $200-$ $300 \mathrm{GHz}$ band, where the atmospheric attenuation is lower than that at $60 \mathrm{GHz}[10]$. Such frequency ranges are very challenging for OEO implementations due to the lack of suitable high-speed modulators. Although polymer-based modulators work beyond $100 \mathrm{GHz}$, problems with their longterm stability remain.

In contrast, an optical frequency comb generator (OFCG) can produce highly phase correlated optical tones over a wide range with a fixed frequency spacing [11] and by filtering two lines of the appropriate frequency spacing, with subsequent heterodyning in a photodiode, it is possible to generate pure mm-wave and $\mathrm{THz}$ signals above $100 \mathrm{GHz}$ [12]. In [13], an OFCG was used to generate a tunable microwave signal in which the frequency tuning was achieved via tuning the bias current of a slave laser that was injection-locked to one of the optical sidebands of the comb. However, tunability was restricted between S-band and Ka-band. Conventionally, in 
OFCGs based on external modulators, a microwave synthesizer is used to provide the seed tone and to determine the frequency spacing. However, it is possible to dispense with an external RF source by driving the OFCG with an OEO instead (either directly or via a self-oscillating approach [14], [15]) for subsequent low-phase noise generation of mm-wave and $\mathrm{THz}$ signals. In [16], a photonic microwave generation technique was demonstrated by using a dual-polarization quadrature phase shifting keying (DP-QPSK) modulator. The upper modulator was biased as a phase modulator to perform the function of an E/O converter for an OEO loop, and in conjunction with a phase-shifted fiber Bragg grating (PSFBG), a microwave photonic filter (MWPF) was also implemented. The electrical signal generated from the OEO loop based on the upper modulator was then used to drive the lower modulator, which was operated as an OFCG. However, only up to five comb lines with a frequency spacing of 12.54 $\mathrm{GHz}$ were observed experimentally, and there is a tradeoff between the power and the mode suppression ratio of the comb.

The self-oscillating OFCG reported in [14] and [15] lacks the frequency tunability of a conventional OEO and hence the $\mathrm{mm}$-wave and $\mathrm{THz}$ signal frequency can only be tuned by changing the selected lines spacing. Recently in [17], we experimentally demonstrated the photonic generation of $\mathrm{mm}$ wave and $\mathrm{THz}$ signals using a tunable OEO driven OFCG. Tunability of the OEO (over $6.58-18.36 \mathrm{GHz}$ ) was achieved through the use of a microwave photonic filter (MWPF)based on the cascade of a phase modulator (PM) and tunable optical bandpass filter(TOBF) [18]; the SSB phase noise of the OEO section is below $-103 \mathrm{dBc} / \mathrm{Hz}$ throughout the entire tuning range. Unlike electrical bandpass filters, for which a narrow passband that can be tuned over several $\mathrm{GHz}$ is difficult to achieve, several MWPF have been demonstrated that can be tuned over several GHz [9][19].

In this paper, we apply the tunable OEO-driven OFCG concept to implement a RoF link in the $\mathrm{THz}$ band for the first time. A key advantage of this system is the ability to generate a tunable low phase noise $\mathrm{THz}$ signal without requiring any reference RF input, hence resulting in a fully microwave photonic implementation which allows direct distribution of signals over optical fiber. Here, an electrical signal from an OEO is used to generate 22 optical comb lines from an OFCG, covering a range of $360 \mathrm{GHz}$ within a $20 \mathrm{~dB}$ envelope. By selecting two optical comb lines, and beating them in a unitraveling carrier photodiode (UTC-PD), we generate $\mathrm{THz}$ signals at $101.5 \mathrm{GHz}$ and $242.6 \mathrm{GHz}$ when the OEO oscillation frequencies are $16.92 \mathrm{GHz}$ and $17.33 \mathrm{GHz}$, respectively. The measured phase noises (after downconversion) of the $101.5 \mathrm{GHz}$ and $242.6 \mathrm{GHz}$ signals are -90 $\mathrm{dBc} / \mathrm{Hz}$ and $-78 \mathrm{dBc} / \mathrm{Hz}$, respectively at $10 \mathrm{kHz}$ offset frequency. To analyse the short-term and long-term stability of the OEO, we also measured the Allan deviation, and the frequency and power drift of the generated RF carrier. An Allan deviation of $6.1 \times 10^{-8}$ is found for a $1 \mathrm{~s}$ observation time at the $17.33 \mathrm{GHz}$ oscillation frequency, while the peak frequency drift is $7 \mathrm{kHz}$, and power fluctuation is below 0.7 $\mathrm{dB}$ over 30 minutes' observation duration.

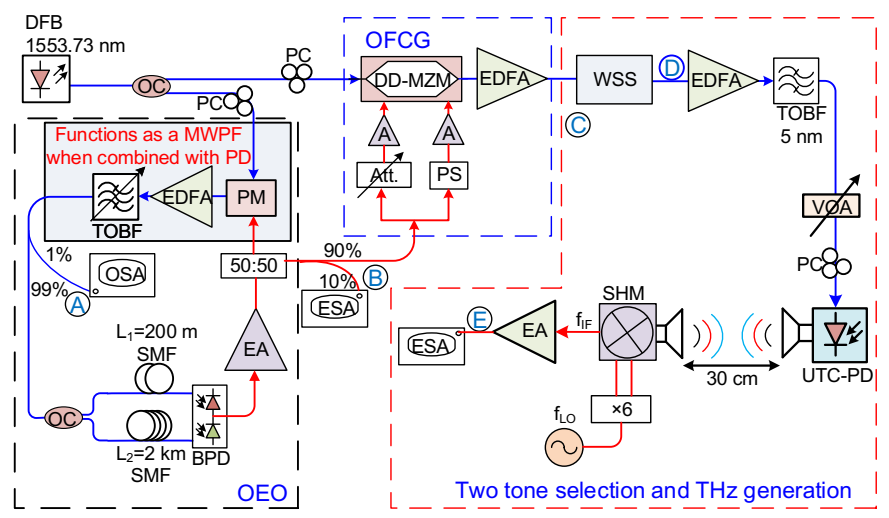

Fig. 1. Experimental arrangement of the OEO-driven OFC generation and $\mathrm{THz}$ wave generation. The blue line represents the optical path while the red line represents the electrical path.

A RoF link is subsequently implemented with the OEO-driven OFCG at the $242.6 \mathrm{GHz}$ carrier frequency and the results are compared with an OFCG driven by an electrical synthesizer. No degradation in performance is observed with the OEOdriven approach. The obtained bit error rate (BER) is below the hard decision forward error correction (HD-FEC) limit $\left(3.8 \times 10^{-3}\right)$ for a wireless distance of $30 \mathrm{~cm}$ and a data rate of $24 \mathrm{Gbps}$ for 16 QAM modulation. These results augur well for the future development of photonics-based $\mathrm{THz}$ radio-overfiber.

The paper is organized as follows. Section II describes the tunable $\mathrm{mm}$-wave and $\mathrm{THz}$ signal generation with the tunable OEO, and the OEO stability is also analysed. A RoF link in the THz band is described in section III. Finally, section IV summarizes and concludes the article.

\section{TUNABLE MM-WAVE AND THz SIGNAL GENERATION}

The experimental arrangement of the proposed concept is shown in Fig. 1. It consists of three subsections: (i) the optoelectronic oscillator (OEO), (ii) the optical frequency comb generator (OFCG), and (iii) two-tone selection and $\mathrm{THz}$ generation section, in which two tones are filtered out, prior to heterodyne detection and transmission over a wireless path. The individual subsections are described in more detail below.

\section{A. MWPF based on a tunable OEO}

A distributed feedback (DFB) laser with a center wavelength of $1553.73 \mathrm{~nm}$ and a linewidth of $10 \mathrm{kHz}$ acts as an optical source (via a 50:50 optical coupler) for both the OEO and OFCG subsections. The phase modulator (PM) within the OEO loop is used as an electrical-to-optical (E/O) converter, in which a modulating signal at $\omega_{m}$ produces anti-phase sidebands around an optical carrier $\left(\omega_{0}\right)$. When combined with a variable bandwidth tunable optical bandpass filter (TOBF), the PM also results in a MWPF [20] (following subsequent optoelectronic conversion in a photodiode), which functions on the principle of asymmetrical sideband suppression as shown in Fig.2(a). Here the center frequency of the optical carrier $\left(\omega_{0}\right)$ is shifted slightly from the center frequency of the optical filter $\left(\omega_{c}\right)$. Photodetection of the optical carrier and the remaining sideband results in a single sideband RF response, where the center frequency and 


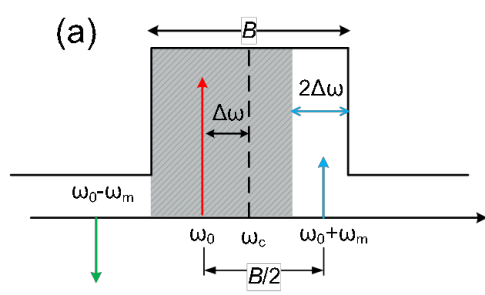

(b)

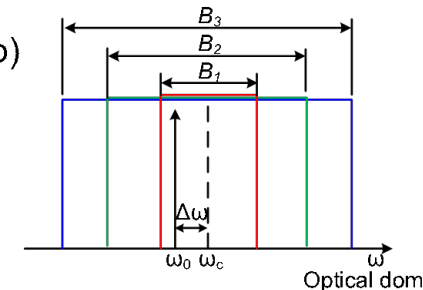

(c) $\quad B=B_{1}=B_{2}=B_{3}$

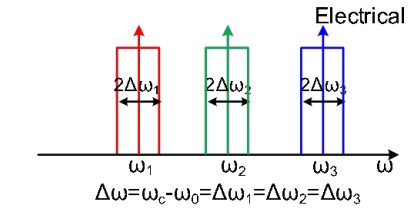

Centre frequency can be tuned from $\omega_{1}$ to $\omega_{3}$ by changing optical filter bandwidth from $B_{1}$ to $B_{3}$
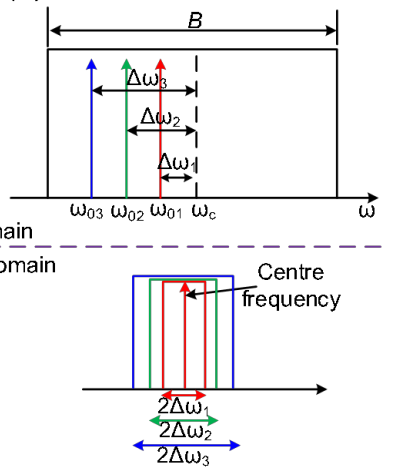

Bandwidth can be tuned from $2 \Delta \omega_{1}$ to $2 \Delta \omega_{3}$ by changing laser oscillation frequency from $\omega_{01}$ to $\omega_{03}$

Fig. 2 (a) Asymmetrically transmitted sidebands (b) center frequency tuning by changing the optical bandwidth $(B)$ of the TOBF, and (c) bandwidth tuning by changing the laser wavelength.

bandwidth are determined by the spectral response of the TOBF. The center frequency in the electrical domain is equal to half the bandwidth of the TOBF $(B / 2)$ while the bandwidth is equal to twice the offset between the laser oscillation frequency and TOBF center frequency $\left(\Delta \omega=\omega_{\mathrm{c}}-\omega_{0}\right)$, as shown in Fig 2(a). By increasing the bandwidth of the TOBF, the center frequency of the RF filter increases $\left(f_{3}>f_{2}>f_{1}\right)$ (Fig.2(b)), while the bandwidth of the RF filter changes with the changes of offset frequency (Fig.2(c)). In the experimental setup, the TOBF is a Yenista Optics XTM-50; this particular model has a minimum and maximum bandwidth of $6.25 \mathrm{GHz}$, and $120 \mathrm{GHz}$, respectively, with a bandwidth resolution of 1 $\mathrm{pm}$ (corresponding to $124.27 \mathrm{MHz}$ for a $1550 \mathrm{~nm}$ wavelength). Therefore, the oscillation frequency of the OEO can be tuned (in principles) from $3.125 \mathrm{GHz}$ to $60 \mathrm{GHz}$ with a step size of $62.13 \mathrm{MHz}$ provided that the oscillation frequency meets the required gain and phase conditions.

The OEO is based on a dual-loop balanced detection configuration in which standard single mode fiber (SMF) lengths of $L_{1}=200 \mathrm{~m}$ and $L_{2}=2 \mathrm{~km}$, respectively, feed a balanced photodiode (BPD) (DSC730, with a 3-dB bandwidth of $25 \mathrm{GHz}$, and a responsivity of $0.60 \mathrm{~A} / \mathrm{W}$ ). We tuned the center wavelength of the TOBF to be as close as possible to the laser oscillation frequency in order to obtain a narrow passband (when used in combination with the dual loop architecture), thus helping to further suppress the side modes. $\mathrm{E} / \mathrm{O}$ and $\mathrm{O} / \mathrm{E}$ conversion losses in the loop are compensated with an erbium doped fiber amplifier (EDFA) in the optical portion and two electrical amplifiers (EA) in cascade in the electrical portion, with the output of this amplifier chain being used to drive both the PM in the OEO loop, and also the DDMZM (Photonic Systems Inc.) in the OFCG.

\section{B. OFC generation}

The microwave output of the OEO passes through a splitter. One of the output arms of this splitter is then connected to an attenuator (Att.) and the other to a phase shifter (PS). Through suitable adjustment of both of these components, the drive signals for the DD-MZM have the required amplitudes and phases so as to satisfy the flat spectrum condition in [21]. The resulting optical comb is then amplified optically prior to being connected to the final section in which two tone selection and $\mathrm{THz}$ frequency generation take place.

\section{Optical two tone selection and THz wave generation}

A programmable wavelength selective switch (WSS) is employed to select two optical tones from the OFCG output that have a wavelength spacing corresponding to the desired $\mathrm{THz}$ frequency, which results from heterodyning in a UTCPD. The WSS was a Finisar WaveShaper (4000S) based on liquid crystal on silicon (LCoS) technology. The optical output of the WSS was then amplified and filtered to remove the amplified spontaneous emission (ASE) noise before photodetection in an unpackaged UTC-PD with an integrated coplanar waveguide (CPW). The optical input power to the UTC-PD was controlled and polarization aligned by a variable optical attenuator (VOA) and a polarization controller (PC) respectively. The output of the photodiode was connected to a $20 \mathrm{dBi}$ horn antenna (WR-5.1) using a coplanar mm-wave probe, with the transmitted $\mathrm{THz}$ signal then being received by an identical horn antenna. In order to measure the phase noise of the received $\mathrm{THz}$ signal, it was down-converted to a microwave intermediate frequency (IF) within the range of RF components by using a sub-harmonic mixer (SHM). A Rohde \& Schwarz synthesizer (SMA100A) was used as a local oscillator (LO), with its output frequency being multiplied ( $\times$ 6) and mixed with the THz signal in the SHM. The IF signal was then amplified and connected to an electrical spectrum analyser (ESA) to measure the phase noise performance.
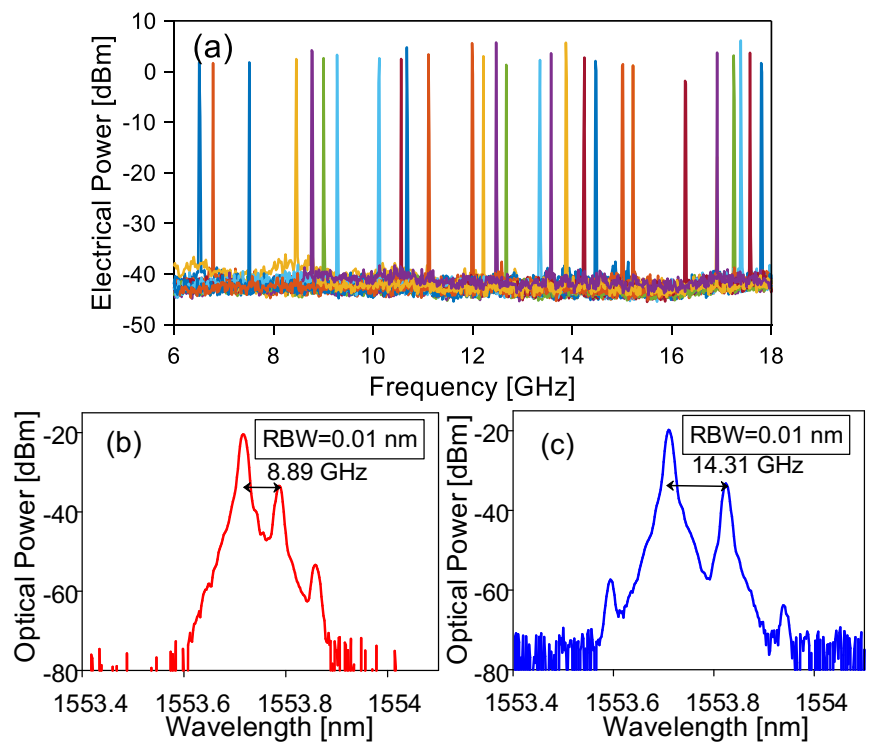

Fig. 3(a) Electrical spectra of the generated microwave signal for different frequencies measured at point $\mathbf{B}$ in Fig.1; (b) and (c) optical spectra at the output of the TOBF for the oscillating frequency $8.89 \mathrm{GHz}$ and $14.31 \mathrm{GHz}$, respectively, measured at point $\mathbf{A}$ in Fig. 1. 


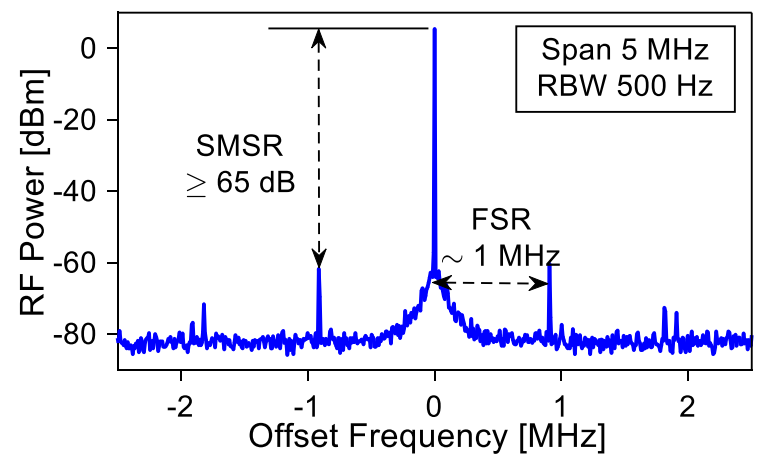

Fig.4 Electrical spectrum of the generated $17.33 \mathrm{GHz}$ signal from the OEO (measured at point $\mathbf{B}$ of Fig.1)

\section{Experiment results}

1) OEO electrical spectrum, phase noise and stability

After the photo-detection process, the PM-TOBF combination functions as a tunable MWPF as described in Section 2.1 II(A); by tuning the bandwidth of the TOBF filter, we were able to generate continuous tunable microwave signals from $6.58 \mathrm{GHz}$ to $18.36 \mathrm{GHz}$. Typical spectra of the generated RF carriers (as measured at point $\mathbf{B}$ in Fig.1) are shown in Fig.3 (a). The optical spectrum at the output of TOBF was measured with an optical spectrum analyzer (OSA) with a resolution bandwidth (RBW) of $0.01 \mathrm{~nm}$ and is shown in Fig. 3 (b) and Fig.3 (c) for OEO oscillation frequencies of $8.89 \mathrm{GHz}$ and $14.31 \mathrm{GHz}$ respectively, in which the wider TOBF bandwidth can be observed for the $14.31 \mathrm{GHz}$ oscillation compared to that at $8.89 \mathrm{GHz}$. The electrical spectrum of the generated RF signal at $17.33 \mathrm{GHz}$ is shown in Fig.4 for a span of $5 \mathrm{MHz}$. Side modes at an offset frequency of $1 \mathrm{MHz}$ from the carrier (corresponding to the short fiber length, $L_{1}=200 \mathrm{~m}$ ) with more than $65 \mathrm{~dB}$ suppression were observed. Side modes at $100 \mathrm{kHz}$ offset corresponding to the long fiber $\left(L_{2}=2 \mathrm{~km}\right)$ were suppressed due to the dual loop effect [22]. We measured the single side band phase (SSB) noise of the OEO and compared it with an external signal generator (R\&S $\left.{ }^{\circledR} \mathrm{SMP}-04\right)$. Here,
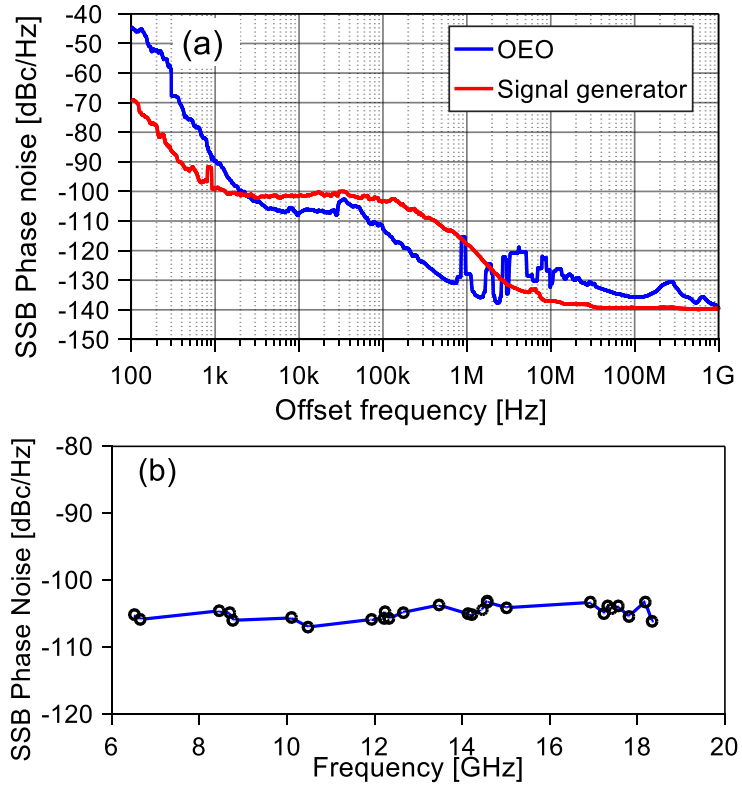

Fig.5. (a) Measured SSB phase noise of the $17.33 \mathrm{GHz}$ oscillation from the OEO (blue line) and R\& ${ }^{\circledR} \mathrm{SMP}-04$ signal generator (red line). (b) $10-\mathrm{kHz}$ phase noise performance as function of oscillating frequency. The SSB phase noise is below $-103 \mathrm{dBc} / \mathrm{Hz} @ 10 \mathrm{kHz}$ offset for the entire tuning range. and in all subsequent phase noise measurements, we used the smoothing option $(1 \%)$ of the spectrum analyzer to display the general trends of the phase noise with offset frequency. At offset frequencies higher than $3 \mathrm{kHz}$ the SSB phase noise of the OEO is better than that of the signal generator. The measured single side band (SSB) phase noise of the OEO and external generator is $-89.5106 \mathrm{dBc} / \mathrm{Hz}$ and $-102 \mathrm{dBc} / \mathrm{Hz}$ respectively at $17.33 \mathrm{GHz}$ for a $10 \mathrm{kHz}$ offset frequency as shown in Fig. 5(a). From the phase noise curve, we also observe side modes at a multiple of $1 \mathrm{MHz}$ offset. The SSB phase noise at $10 \mathrm{kHz}$ offset frequency of the generated electrical signals is shown in Fig. 5(b), and was found to be below $-103 \mathrm{dBc} / \mathrm{Hz}$ for the whole tuning range. This is in agreement with other results which indicate that the phase noise of OEO does not depend on the oscillation frequency [7] [23].

We then measured the Allan deviation $\left(\sigma_{\tau}\right)$ to determine the short time stability of the OEO. Figure 6(a) shows the measured Allan deviation, as calculated using the Allan deviation measurement option of the Rohde \& Schwarz spectrum analyzer (FSU) with the frequency counter method. The measured Allan deviation for an observation time of 1 second is $6.1 \times 10^{-8}$ for the oscillation frequency of $17.33 \mathrm{GHz}$
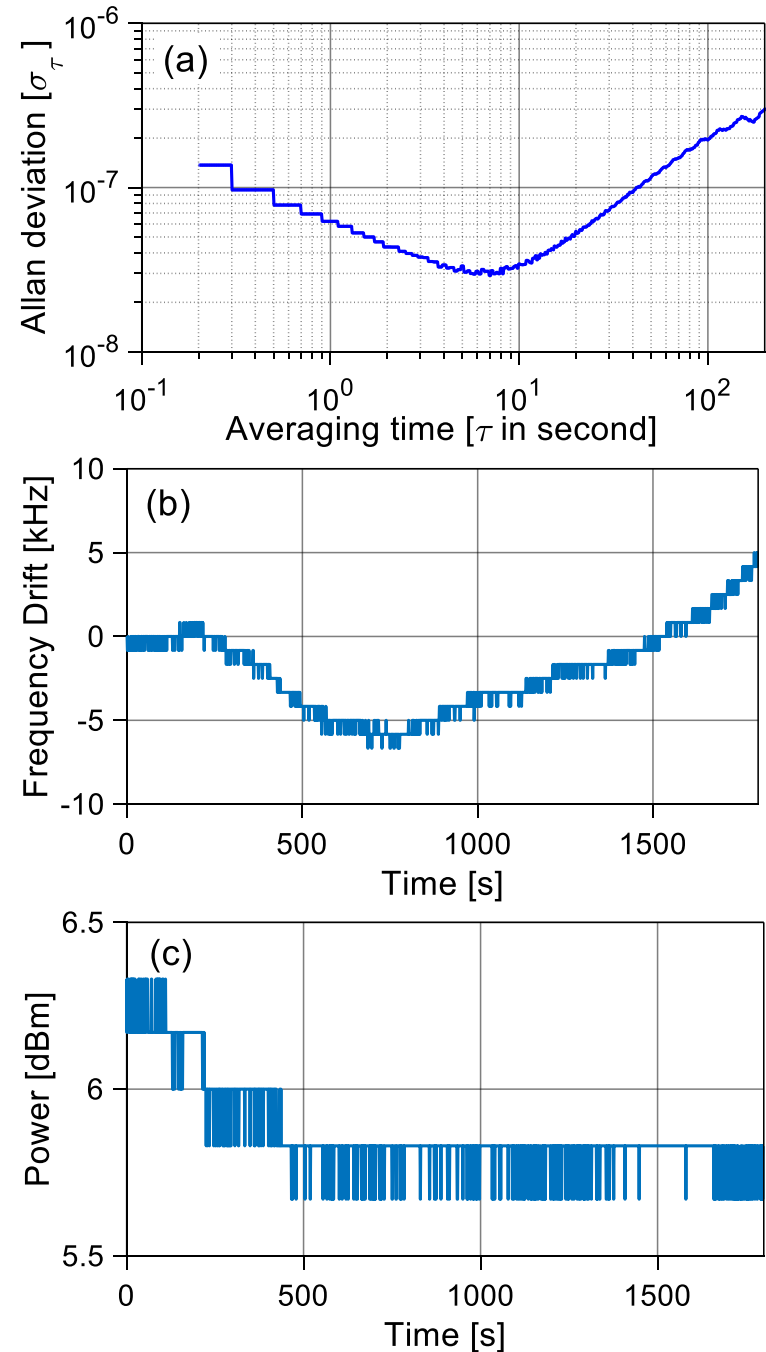

Fig.6. (a) Allan deviation of the generated $17.33 \mathrm{GHz}$ signal, (b) frequency drift over time, (c) power drift over time. 
meaning there is approximately a $1 \mathrm{kHz}$ rms frequency movement between the two observations that are 1 second apart. The shape of this plot is in line with that observed in the literature [24], with the initial decrease being due to flicker phase and white phase modulation noise, and the subsequent increase due to random walk FM and linear frequency drift. We also measured the oscillation frequency and electrical output power of the OEO at one second intervals for half an hour using a spectrum analyzer. Fig.6(b) shows the frequency drift and Fig.6(c) shows the oscillation power. The peak frequency drift is $7 \mathrm{kHz}$ and the power deviation is less than $0.7 \mathrm{~dB}$ during the measurement duration. The step-like behaviour in Fig.6 is due to the spectrum analyzer being programmed to monitor and store the frequency of the oscillation and oscillation power after every second, hence due to the relatively high resolution bandwidth, any small changes between steps were not monitored.

2) Generated mm-wave and $\mathrm{THz}$ waves

It is possible to generate $\mathrm{THz}$ signals over a broad frequency range by varying either the OEO oscillation frequency or filtering out two tones from the comb that are separated by an integer multiple of the comb line spacing. The optical comb spectra for three different OEO oscillation frequencies (10.5 $\mathrm{GHz}, 16.92 \mathrm{GHz}$, and 17.33 GHz) are shown in Fig.7 (a), (b)

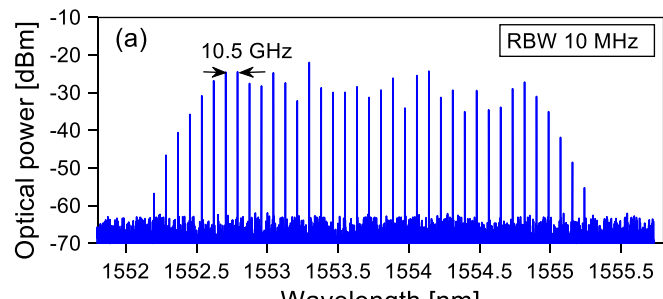

Wavelength [nm]
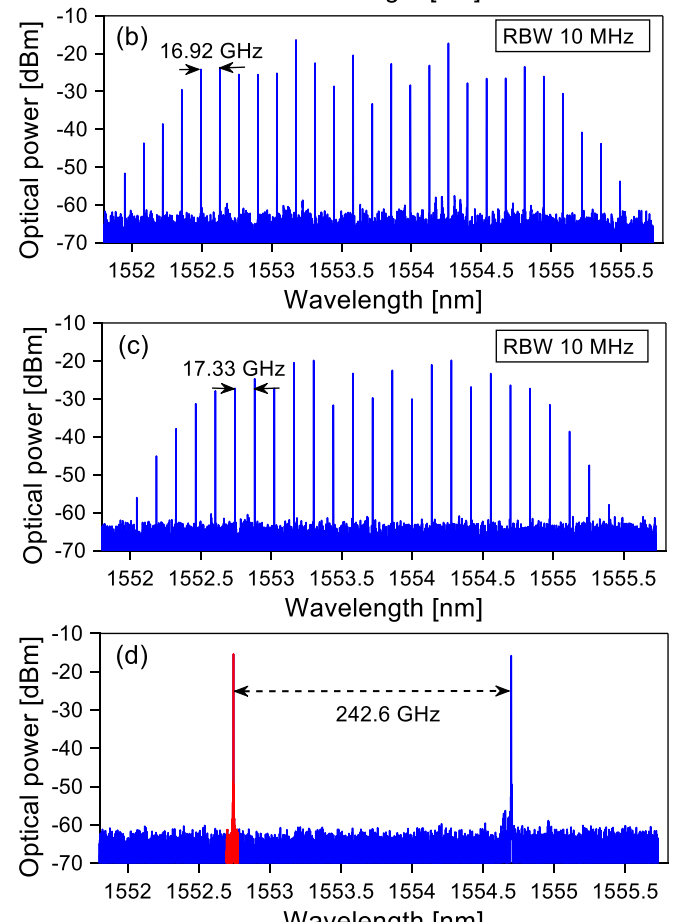
Wavelength [nm]

Fig.7. Optical spectra of the generated optical frequency combs with a frequency spacing of (a) $10.5 \mathrm{GHz}$ (b) $16.92 \mathrm{GHz}$ (c) $17.33 \mathrm{GHz}$ at the center wavelength of $1553.73 \mathrm{~nm}$ (measured with an OSA at point $\mathbf{C}$ in Fig.1; (d) Optical spectrum of the selected comb lines (measured with an OSA at point $\mathbf{D}$ in Fig. 1).
\& (c), respectively. A spectrum of 22 optical tones with a spacing of $17.33 \mathrm{GHz}$ is observed (Fig.7(c)), covering a bandwidth of $360 \mathrm{GHz}$ within a $20 \mathrm{~dB}$ envelope. In this work, comb flatness was not prioritized since we focused primarily on generation of mm-wave and $\mathrm{THz}$ signals by two tone selection (as described below) and implementation of an RoF link at $\mathrm{THz}$ band (as will be discussed in section III). However, it is possible in principle to improve the comb flatness as in [21].By selecting any two optical comb lines, it is possible in principle to generate $\mathrm{THz}$ signals up to $360 \mathrm{GHz}$ through heterodyne detection with a UTC-PD. In this work, we generated an RF signal at $101.5 \mathrm{GHz}$ (for two lines spaced six intervals apart with spacing of $16.9 \mathrm{GHz}$, i.e. $N=6$ ), and $242.6 \mathrm{GHz}$ (for two lines spaced 14 intervals apart with a spacing of $17.33 \mathrm{GHz}$, i.e. $N=14$ ). Fig. 7(d) shows the optical spectra of the selected two optical tones for the latter case. The electrical spectra of the down-converted signals are shown in Fig. 8(a) and 8(b) for the $101.5 \mathrm{GHz}$ and $242.6 \mathrm{GHz}$ signal, respectively. We found $\sim 1 \mathrm{MHz}$ spaced side modes (corresponding to the short length $L_{1}=200 \mathrm{~m}$ ) around the carrier and $\sim 100 \mathrm{kHz}$ spaced side modes (corresponding to the long length $L_{2}=2 \mathrm{~km}$ ) with relatively low amplitude around the $\sim 1 \mathrm{MHz}$ spaced side modes. The SSB phase noise of the down-converted signals was measured and is shown in Fig.8(c) and 8(d). The SSB phase noise of the $101.5 \mathrm{GHz}$ and $242.6 \mathrm{GHz}$ signals is $-90 \mathrm{dBc} / \mathrm{Hz}$ and $-78 \mathrm{dBc} / \mathrm{Hz}$ respectively at $10 \mathrm{kHz}$ offset frequency; the SSB phase noise is degraded by $16 \mathrm{~dB}$ for the $101.5 \mathrm{GHz}$, and $26 \mathrm{~dB}$ for the $242.6 \mathrm{GHz}$ signal from their seed oscillation given that the SSB phase noise of $16.9 \mathrm{GHz}$ and $17.33 \mathrm{GHz}$ signals are $-106 \mathrm{dBc} / \mathrm{Hz}$ and $-104 \mathrm{dBc} / \mathrm{Hz}$ respectively at $10 \mathrm{kHz}$ offset. This
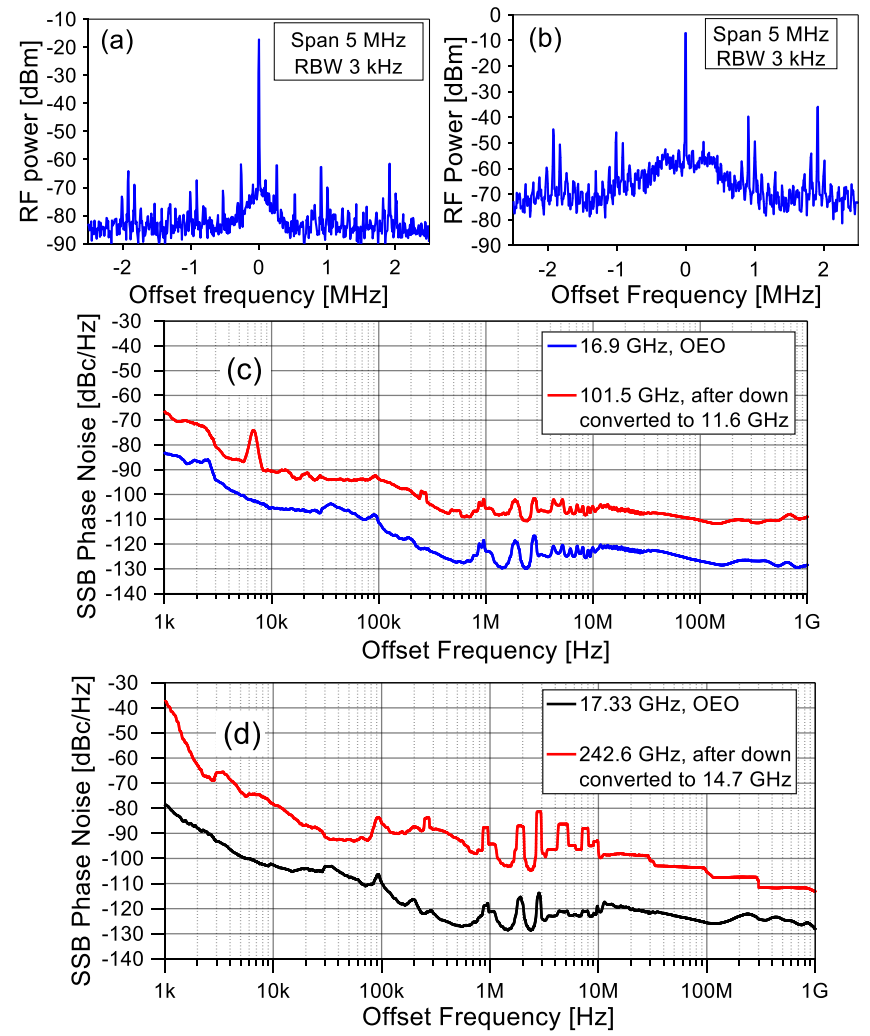

Fig. 8. Electrical spectra of the down-converted THz signals (a) $101.5 \mathrm{GHz}$, and (b) $242.6 \mathrm{GHz}$. Measured SSB phase noise of the down-converted $\mathrm{THz}$ signals (c) $101.5 \mathrm{GHz}$, and (d) $242.6 \mathrm{GHz}$ (measured at point $\mathrm{E}$ in Fig.1). 


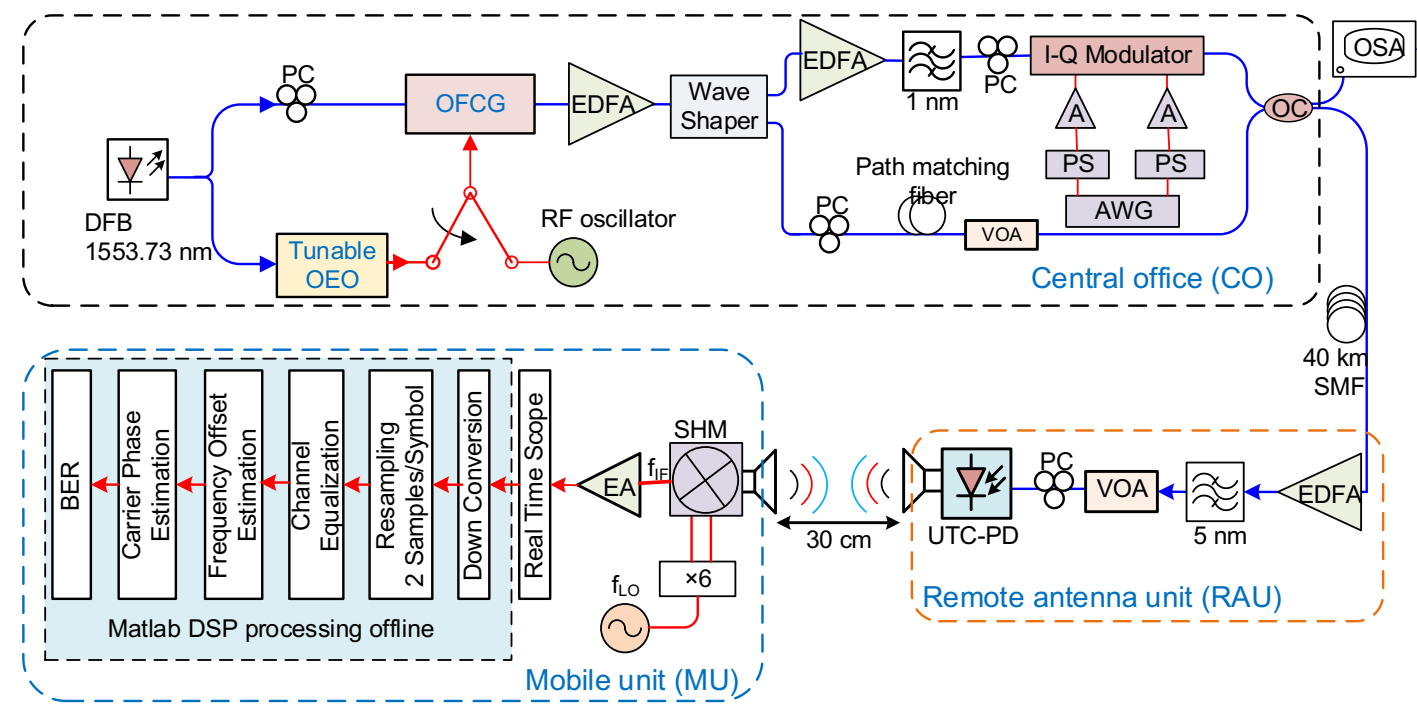

Fig.9. Experimental set-up of the tunable OEO driven OFCG based radio over fiber link. The optical and electrical paths are represented by the blue and red lines respectively

degradation also includes the phase noise addition during the down-conversion process due to the optical and electrical amplifiers, which equates to a $3 \mathrm{~dB}$ contribution for the 242.6 $\mathrm{GHz}$ signal. The trend of the SSB phase noise degradations is in good agreement with the carrier phase noise generated by the multiplication $\left(20 \times \log _{10} N\right)$.

\section{ROF LINK BASED ON TUNABLE OEO DRIVEN OFCG}

We developed a RoF link at $242.6 \mathrm{GHz}$ using the OEO-driven OFCG. Fig.9 shows the experimental arrangement for the RoF link, which uses the OEO and OFCG subsections that were described in sections 2.1 and 2.2 respectively. To implement the RoF link, the output of the OFCG section was passed through a WSS after optical amplification via an EDFA. The OEO oscillation frequency was set to $17.33 \mathrm{GHz}$ (with the corresponding optical comb as shown in Fig.7 (c)). Two optical tones with a frequency spacing of $242.6 \mathrm{GHz}$ were selected and guided into two different output ports of the WSS; one optical tone was used as a local oscillator (LO) while the other was used for data modulation. This second tone was amplified by an EDFA and passed through a $1 \mathrm{~nm}$ optical band pass filter to eliminate the out of band amplified stimulated emission (ASE) noise. The output of the filter was then fed to an I-Q modulator after being polarization controlled. A Tektronix AWG7000 arbitrary waveform generator (AWG) with a sampling rate of $50 \mathrm{GSa} / \mathrm{s}$ was used to generate $2^{11}-1$ pseudo random bit pattern (PRBS) I and Q signals. Prior to being connected to the I-Q modulator, the I and Q signals were electrically amplified and phase shifted. During the experiment, we used 10 Gbaud QPSK and 6 Gbaud 16 QAM modulation format with a square root raised cosine (SRRC) filter of 0.1 .

The LO tone was passed through a polarization controller (PC) to align the polarization state and a short path matching fiber of $39.5 \mathrm{~m}$ length was used to compensate for the optical path difference introduced between the two branches (modulator and LO branch) due to the inclusion of the EDFA and optical filter in the modulator branch. An optical attenuator (OA) was used in the LO branch to match the optical power between the modulated and LO tone. The power ratio of the optical carrier to the modulated signal affects the signal quality as follows: high optical carrier-to-signal ratios result in ASE noise being dominant and increase the BER, while for low optical carrier-to-signal ratios the SNR of the heterodyne signal at the RF carrier is reduced. The optimum value occurs for an optical carrier-to-signal power ratio of 0 $\mathrm{dB}$ [25]. The modulated tone and $\mathrm{LO}$ tone were then combined in an optical coupler (OC), one output of which was connected to an optical spectrum analyzer (OSA) while the other was passed through a SMF of $40 \mathrm{~km}$ length. The optical spectrum after combining the two tones is shown in Fig.10 (a). Prior to detection with an unpackaged UTC-PD, the optical signal was amplified with an EDFA to compensate the fiber transmission loss, filtered with a $5 \mathrm{~nm}$ OBPF to remove out-of-band ASE, controlled with a variable optical attenuator (VOA) to evaluate the system performance, and polarization aligned with a PC to reduce polarization dependent loss. The beating product of the
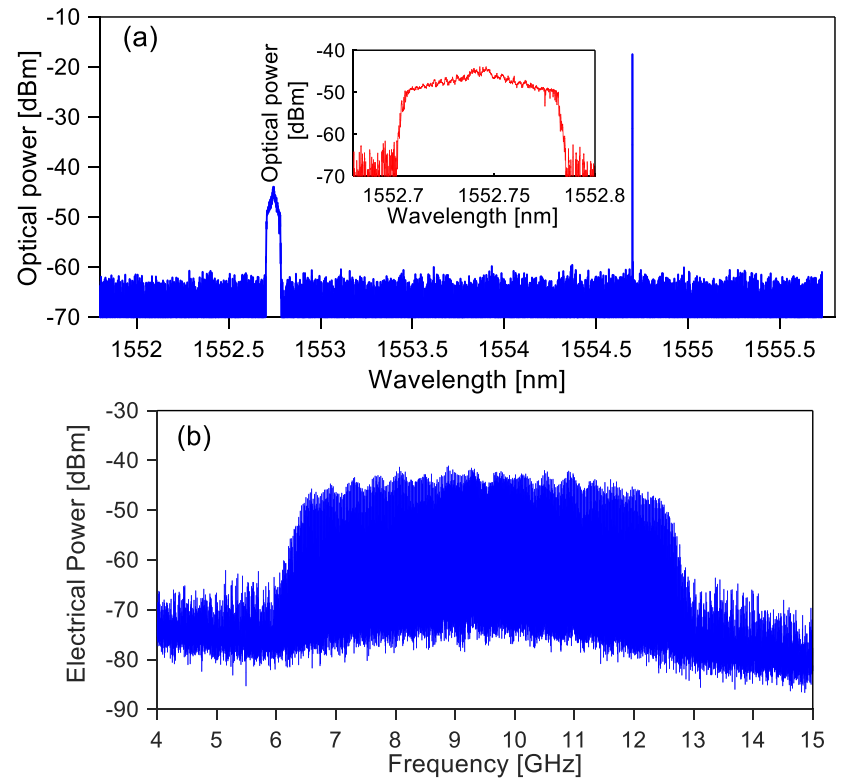

Fig.10. (a) Optical spectrum of the selected two tone for RoF link. The lefthand tone is modulated with 10 Gaud QPSK. The inset shows the zoom view of data modulation. (b) Electrical spectrum using FFT of the received IF signal for 6 Gbaud 16 QAM. 


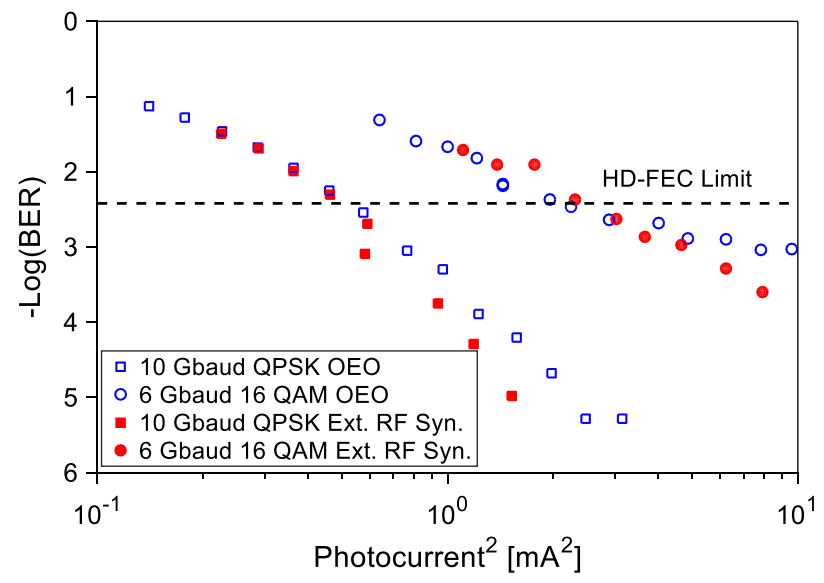

Fig.11. BER as a function of photocurrent.

LO and modulated tone in the UTC-PD was transmitted over a wireless distance of $30 \mathrm{~cm}$ with a $20 \mathrm{dBi}$ horn antenna. Another antenna of the same gain and polarization received the transmitted $\mathrm{THz}$ signal, which was subsequently downconverted to an IF frequency with the electrical LO-SHM combination as described in section $\mathrm{II}(\mathrm{C})$. The electrical LO signal frequency was selected in such a way so as to place the IF signal around $9 \mathrm{GHz}$, within the bandwidth of the analog to digital converter (ADC) of the real time oscilloscope (RTO). The IF signal was then amplified and connected to a RTO with $36 \mathrm{GHz}$ bandwidth and sampling rate of $80 \mathrm{GSa} / \mathrm{s}$ for further processing; the IF spectrum of the down-converted $\mathrm{THz}$ signal obtained by fast Fourier transform (FFT) in the RTO is shown in Fig.10(b). Due to the non-flat response of the IF amplifier, the IF signal has a slight asymmetry.

The digitized signal was then processed with an offline DSP processor in MATLAB to demodulate the QPSK/ QAM signal. The DSP processing includes down-conversion, resampling (2 samples/symbol), channel equalization (blind equalizer algorithm), carrier recovery and phase noise estimation. As stated earlier, the received RF signal was captured by a digital sampling scope at $80 \mathrm{GSa} / \mathrm{s}$. The recorded samples were reduced to 2 samples per symbol to reduce the calculation time taken for the DSP algorithms [26]. Finally, the bit error rate (BER) performance was evaluated by using a VOA to vary the input optical power to the UTC-PD.

The RoF link was evaluated with two different modulation formats: 10 Gbaud QPSK and 6 Gbaud 16 QAM. The BER was measured first when the OFCG was driven by the OEO, then when the OFCG was driven by an external RF synthesizer. Fig. 11 shows the measured BER versus received optical power for the four experimental conditions. There is a small power penalty between the OEO-driven and external synthesizer driven curve which is due to the effect of phase noise. The phase noise and drift of the RF carrier based on the OEO method dominates over the long sequence of the data in Fig. 11. This can be improved as will be mentioned in the conclusions. The external RF synthesizer has superior close to carrier phase noise performance to the OEO as shown in Fig.5(b). The obtained BER is below the hard decision forward error correction limit of $3.8 \times 10^{-3}$ for $7 \%$ overhead. The obtained data rates are for $20 \mathrm{Gbps}$ and $24 \mathrm{Gbps}$ for 10 Gbaud QPSK and 6 Gbaud 16 QAM respectively.

\section{CONCLUSION}

A low phase noise $\mathrm{THz}$ generation using a tunable OEO and an OFC generator was demonstrated. With the OEO oscillation frequency set to $17.33 \mathrm{GHz}$, a $242.6 \mathrm{GHz}$ signal with a phase noise level of $-78 \mathrm{dBc} / \mathrm{Hz}$ at $10 \mathrm{kHz}$ offset was obtained. This was then used to implement a RoF link which was evaluated for wireless transmission of 10 Gbaud QPSK and 6 Gbaud 16 QAM over $40 \mathrm{~km}$ of SMF and a $30 \mathrm{~cm}$ wireless link. Through tuning the OEO oscillation frequency, the demonstrated system can generate a tunable $\mathrm{THz}$ signal. Hence it can potentially be applied to multi-carrier or multiband radio over fiber links with a reconfigurable $\mathrm{THz}$ carrier frequency.

The generated $\mathrm{THz}$ signal relies on the stability of the OEO. The following points could be considered for further improvement in the system performance.

- Although the SSB phase noise of our OEO is comparable to other reported designs, the SSB phase noise of the system could be improved using a longer fiber loop. In addition, the long-term stability of the set-up is affected by thermal stabilization of the fiber. The refractive index of the fiber depends on temperature, hence temperature variation results in frequency drift of the OEO oscillation. This oscillation frequency drift may be controlled by using a stabilized temperature box or by phase locking with an external reference.

- Hollow core photonic band gap (HC-PBG) fibers have been recently considered in OEO implementations to enhance long term stability, and improve the temperature stability by a factor of more than 15, as compared to SMF implementations [27].

- As mentioned earlier, the close to carrier phase noise depends on the Q-factor of the cavity. A high-Q cavity also helps to improve the SMSR, but a single band pass MWP filter is incapable of providing a high Q. A cascaded MWPF can be a potential solution where a single passband MWPF could, for example, be cascaded with an optoelectronic infinite impulse response section [28].

\section{REFERENCES}

[1] A. Kanno, P. T. Dat, N. Sekine, I. Hosako, and T. Kawanishi, "High-Speed Coherent Transmission Using Advanced Photonics in Terahertz Bands," no. 12, pp. 1071-1080, 2015.

[2] A. Kanno et al., "Seamless fiber-wireless bridge in the millimeterand terahertz-wave bands," J. Light. Technol., vol. 34, no. 20, pp. 4794-4801, 2016.

[3] A. Kanno et al., "Coherent radio-over-fiber and millimeter-wave radio seamless transmission system for resilient access networks," IEEE Photonics J., vol. 4, no. 6, pp. 2196-2204, 2012.

[4] A. Kanno, P. T. Dat, N. Yamamoto, and T. Kawanishi, "Millimeterwave radio-over-fiber network for linear cell systems," J. Light. Technol., vol. 36, no. 2, pp. 533-540, 2017.

[5] J. A. Nanzer, P. T. Callahan, M. L. Dennis, and T. R. C. Jr, "Photonic Signal Generation for Millimeter- Wave Communications," Johns Hopkins APL Tech. Dig. (Applied Phys. Lab., vol. 30, no. 4, pp. 299-308, 2012.

[6] A. J. Seeds, "TeraHertz photonics for communications," J. Light. Technol., vol. 33, no. 3, pp. 579-587, 2014.

[7] X. S. Yao and L. Maleki, "Optoelectronic microwave oscillator," $J$. Opt. Soc. Am. B, vol. 13, no. 8, pp. 1725-1734, Aug. 1996.

D. Eliyahu, D. Seidel, and L. Maleki, "RF amplitude and phase- 
noise reduction of an optical link and an opto-electronic oscillator," IEEE Trans. Microw. Theory Tech., vol. 56, no. 2, pp. 449-456, 2008.

[9] H. Peng et al., "Tunable DC-60 GHz RF generation utilizing a dualloop optoelectronic oscillator based on stimulated brillouin scattering," J. Light. Technol., vol. 33, no. 13, pp. 2707-2715, 2015.

[10] G. Ducournau et al., "THz Communications using Photonics and Electronic Devices: the Race to Data-Rate," J. Infrared, Millimeter, Terahertz Waves, vol. 36, no. 2, pp. 198-220, 2015.

[11] H. Shams et al., "Coherent frequency tuneable thz wireless signal generation using an optical phase lock loop system," in MWP 2017 2017 International Topical Meeting on Microwave Photonics, 2017, vol. 2017-Decem, pp. 2-5.

[12] S. Koenig et al., "Wireless sub-THz communication system with high data rate," Nat. Photonics, vol. 7, pp. 977-981, 2013.

[13] W. T. Wang, J. G. Liu, W. H. Sun, W. Chen, and N. H. Zhu, "Multiband local microwave signal generation based on an optical frequency comb generator," Opt. Commun., vol. 338, pp. 90-94, Mar. 2015.

[14] G. K. M. Hasanuzzaman, A. Kanno, P. T. Dat, and S. Iezekiel, "Wband radio-over-fiber link based on self-oscillating optical frequency comb generator," in Optical Fiber Communication Conference, 2018, p. paper W1F.6.

[15] G. K. M. Hasanuzzaman, A. Kanno, P. T. Dat, and S. Iezekiel, "Self-oscillating optical frequency comb: Application to low phase noise millimeter wave generation and radio-over-fiber link," $J$. Light. Technol., vol. 36, no. 19, pp. 4535-4542, 2018.

[16] Y. Chen, S. Liu, and S. Pan, "Multi-format signal generation using a frequency-tunable optoelectronic oscillator," Opt. Express, vol. 26, no. 3, p. 3404, Feb. 2018.

[17] G. K. M. Hasanuzzaman, H. Shams, C. C. Renaud, J. C. Mitchell, and S. Iezekiel, "Photonic $\mathrm{THz}$ Generation using Optoelectronic Oscillator-driven Optical Frequency Comb Generator," in 2018 International Topical Meeting on Microwave Photonics (MWP),
2018, pp. 1-4.

[18] X. Xie et al., "Widely Tunable Dual-loop Optoelectronic Oscillator," Cleo 2014, no. c, p. STu2G.2, 2014.

[19] C. Li et al., "Widely tunable optoelectronic oscillator using a dispersion-induced single bandpass MPF," IEEE Photonics Technol. Lett., vol. 30, no. 1, pp. 7-10, 2018.

[20] T. Chen, X. Yi, L. Li, and R. Minasian, "Single passband microwave photonic filter with wideband tunability and adjustable bandwidth.," Opt. Lett., vol. 37, no. 22, pp. 4699-701, 2012.

[21] T. Sakamoto, T. Kawanishi, and M. Izutsu, "Asymptotic formalism for ultraflat optical frequency comb generation using a MachZehnder modulator.," Opt. Lett., vol. 32, no. 11, pp. 1515-1517, 2007.

[22] X. S. Yao and L. Maleki, "Multiloop optoelectronic oscillator," IEEE J. Quantum Electron., vol. 36, no. 1, pp. 79-84, 2000.

[23] L. Maleki, "The opto-electronic oscillator (OEO): Review and recent progress," in 2012 European Frequency and Time Forum, 2012, pp. 497-500.

[24] E. Rubiola, Phase noise and frequency stability in oscillators. Cambridge: Cambridge University Press, 2008.

[25] S. Jansan, I. Morita, and H. Tanaka, "Carrier-to-signal power ratio in fiber-optic SSB-OFDM transmission systems," in ICICE General conference, 2007, p. 363.

[26] H. Shams, M. J. Fice, K. Balakier, C. C. Renaud, F. van Dijk, and A. J. Seeds, "Photonic generation for multichannel $\mathrm{THz}$ wireless communication.," Opt. Express, vol. 22, no. 19, pp. 23465-72, 2014.

[27] U. S. Mutugala et al., "Optoelectronic oscillator incorporating hollow-core photonic bandgap fiber," Opt. Lett., vol. 42, no. 13, pp. 2647-2650, 2017.

[28] A. Liu et al., "Spurious Suppression in Millimeter-Wave OEO with a High-Q Optoelectronic Filter," IEEE Photonics Technol. Lett., vol. 29, no. 19, pp. 1671-1674, 2017. 